\title{
Geo-Team Formation Model for Impromptu Activities
}

\author{
Maryam mahdavyRad \\ School of Computer Science, University of Windsor, Windsor, ON, Canada \\ mahdavy@uwindsor.ca
}

Introduction In Team Formation Problem (TFP), considering an undirected and unweighted graph $\mathrm{G}=(\mathrm{V}, \mathrm{E})$, we want to identify densely connected users $(\mathrm{V})$ as the team members who match given specifications for various teams [1]. Geo-social networks can be modelled as a social graph $\mathrm{G}=(\mathrm{V}, \mathrm{E})$, which involves user's spatial information. An example of Geo social network is shown in Figure 1. Finding teams in geo-social networks aims to create socially cohesive and spatially close team members, which should simultaneously satisfy the team's required skills [2]. Impromptu activities such as health supporting team arrangements require socially and spatially close experts who quickly deploy to teams. In this problem, the multiple team's required features make the team formation problem a challenging problem.

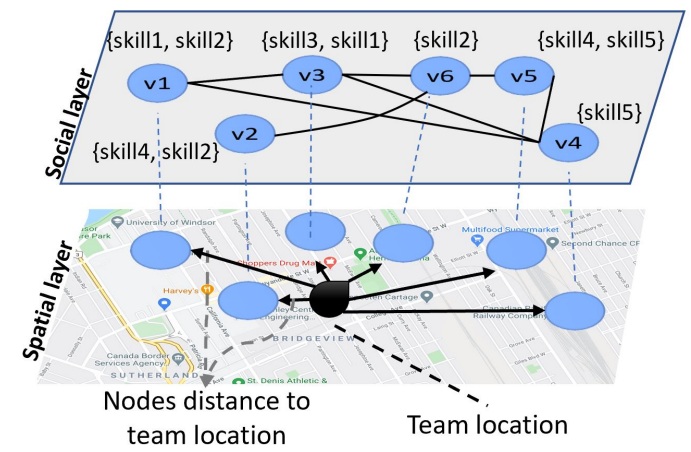

Figure 1. An example of a Geo-Social Network

Team formation problem considering spatial constraint has drawn many recent research interests. The first method that has been applied to this problem is considering the nearest neighbour query to find spatially close team members [3, 4]. Following researches tackle the team formation problem between friends, which are considered strong friendship relations based on social cohesiveness [5]. Recently, Lappas et al. [6] apply skills constraints while considering the team's social cohesiveness. Recently, Shen et al.[7] propose the SSTQ model, which covers the spatial, social and skills constraints, but the number of required skills is one, plus they do not consider the contribution capacity and skill weights as well. Moreover, Chen et al. [8] provide the MKCSSG model that considers spatial, social and skills constraints for team formation. Still, their social cohesiveness approach does not update the social metric for subgraphs cohesively. To fill this gap, we use the p-core-truss method [9], which recognizes more cohesive subgraphs. Also, MKCSSG ignores skill weights in which could be considered as years of experience (YE). Skill weights allow our Geo-team formation model that between two users with 1 (YE) and 10 (YE) choose the user with higher experience and skill weights.

In the literature, There are different methods to dedicate tasks. The Coalition formation [10] in multi-agent systems is also related to this problem [11]. However, the Coalition formation in a multi-agent system is based on selection agents from available groups[12]. A team and a coalition both are a group of cooperative users who follow the same goal.

*corresponding_mahdavy@uwindsor.ca

This article is (C) 2021 by author(s) as listed above. The article is licensed under a Creative Commons Attribution (CC BY 4.0) International license (https://creativecommons.org/licenses/by/4.0/legalcode), except where otherwise indicated with respect to particular material included in the article. The article should be attributed to the author(s) identified above. 
Nevertheless, team members are more dependent on each other. Moreover, Cooperation is more common among team users than coalitions. So the usage of coalition formation or team formation is based on the problem definition and how the cohesive relation between members is required. Furthermore, as indicated, the base network that is indicated for creating the team or coalition [13][14]. Also, In most coalition formation researches in literature, all the constraints like spatial, social and textual constraints are not considered because it increases computational complexity significantly [15].

\section{Research Problem}

In the Geo-team formation, the main problem is creating the timely invitation in large geo-social networks to the groups of users that are highly matched with the required list of skills with highest skill's weight while socially and spatially is satisfied with the team constraints. The Geo-social networks data and team's constraints formally are described below.

Geo-social networks data. An undirected unweighted geo-social graph $\mathrm{G}=(\mathrm{V}, \mathrm{E})$ where $\mathrm{V}$ indicated the nodes (users) of the graph $v_{i} \in$ Vwherev $_{i}, i=1,2, . ., m$ and $\mathrm{E}$ presented the list of edges $e_{i} \in$ Ewheree $_{i}, i=1,2, . ., n$. Each edge show the relation between two nodes $u_{i}, v_{i} \in V$, for example friendship relation. Each node $\mathrm{v}$ posses a location which show the latitude and longitude of each user on a two dimensional plain $(\mathrm{x}, \mathrm{y})$ and a list of skills $\mathrm{K} k_{j} \in K$ with a weight for each skills $w_{j}$.

Team's constraints information. The team's constraints include required skills N, number of required skills for each skill $\mathrm{O}$ (a set of integers), contribution capacity k (an integer), the social constraints c (an integer), and the team location t (latitude and longitude), for example, team1 query= required skills $\mathrm{N}$ (architecture, project manager), number of required skills $\mathrm{O}:(3,2)$, contribution capacity k:2, social constraint c: 5 , team location $\mathrm{t}:(1.4,3.5)$.

Based on geo-social network data and team's constraint information, the problem is to effectively find an optimal solution to assemble teams $T_{i}$ of users $(\mathrm{V})$ that are socially cohesive, and spatially close to the team location t, and contain indicated number of required skills for each task ( $p_{i}$ considering the user' weights of skills and the team contribution capacity $\mathrm{k}$.

\section{Proposed Solution and Approach}

To tackle the Geo-team formation problem, We propose a three-step solution. In the first two steps, we create a framework that extremely prunes the search space based on (1) spatial distance pruning based on nodes euclidean distance to the team location [16] and (2) social cohesiveness pruning using c-core-truss [9]. Moreover, in the third step, based on the created framework, a maximum flow network is established and considers users with required skills maximum skill weights.

Step1. spatial distance pruning based on nodes euclidean distance. In this step, we want to decrease the number of nodes that should be evaluated by pruning the graph data based on social distance of nodes to the team location t. For this purpose, we create a disjoint set data structure and skills summation list from the input graph, which enables us to quickly traverse each subgraph $\mathrm{H}=(\mathrm{v}, \mathrm{E})$ in $\mathrm{o}(|\mathrm{E}(\mathrm{H})|)[17]$. We start creating the disjoint data structure based on the edge list, which is sorted in non-increasing order based on their Euclidean distance to the team location t. We stop creating subgraphs when one of the subgraph's skill summation list satisfy the required team skill.

Two functions are used for creating the disjoint set data structure: union and find. The union function connects two nodes and puts them in a subgraph if they are not in the same 


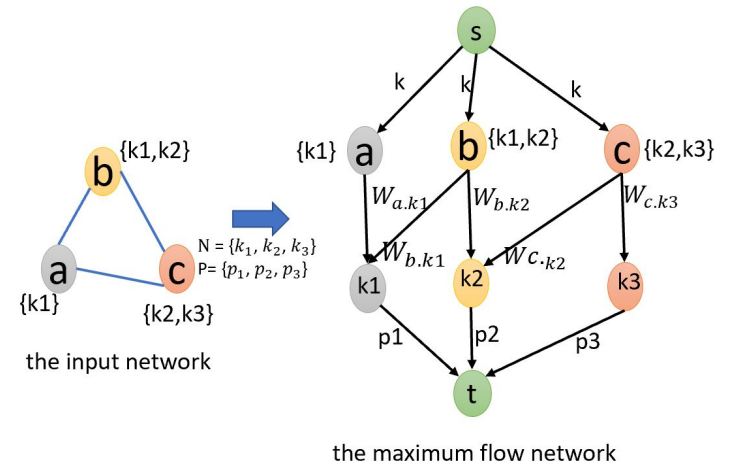

Figure 2. creating a maximum flow network

subgraph. Moreover, the find function indicates if there is any connecting path between two nodes. In this step, after every union function, we add the nodes skills to that subgraph skills summation list. Furthermore, if the summation list of each subgraph meets the team required skill list, we stop this step.

Step2. social cohesiveness pruning using c-core-truss To check the social cohesiveness, we check c-core-truss [9] just on subgraphs which their summation list of skills is satisfied the team list of skills. This method bound the number of c-core-truss checking algorithms. Given a subgraph $\mathrm{H} \subseteq \mathrm{G}$, a parameter $\propto \geq 0$ and an integer $p \geq 2$, a p-coretruss $\mathrm{H}$ is the maximal subgraph of $\mathrm{G}$, in which each edge e satisfies $\operatorname{degsup}_{H}(e) \geq k$ Where $\operatorname{degsup}_{H}(e)=\max \left\{\sup _{H}(e)+2, \propto\right.$.degree $\left.(e)\right\}$. And $\boldsymbol{s u p}_{H}(e)=$ the number of triangles containing $e=\left|\left\{\triangle_{u v w}:(u, w),(v, w) \in E(H)\right\}\right|$. And degree $(e)=\min \{$ degree $(\mathrm{u})$, degree(v)\} [9].

Step3.creating a maximum flow network Directed flow network. The flow network considered in this step is a directed graph $\mathrm{N}=(\mathrm{V}, \mathrm{E})$ with a set of nodes $\mathrm{V}$ and directed edge set $\mathrm{E}$, having a unique source node $\mathrm{s}$, a unique sink node $\mathrm{t}$, and a non-negative capacity $\mathrm{c}(\mathrm{u}, \mathrm{v})$ for every directed edge $(\mathrm{u}, \mathrm{v})$. Maximum weight skills checking is utilized to check if a graph contains the minimum required list of skills besides checking the highest skill weight for a team formation task considering the contribution capacity. Given a flow network $\mathrm{S}$ $=(\mathrm{V}, \mathrm{E})$ and partition its nodes into $\mathrm{S}$ and $\mathrm{T}$ parts, the sum of $\mathrm{S}$ and $\mathrm{T}$ capacities is minimized. The flow network $\mathrm{S}$ is created based on the list of required skills $\mathrm{N}$, the number of needed skills $\mathrm{P}$, contribution capacity r, and user weight's skills $\mathrm{W}$ of subgraph $\mathrm{S}$. For each vertex node $n \in V(N)$, an edge from $s$ to $n$ with the capacity of $r$ is created. For each skill node representing skill, an edge from the skill node to the t node with a capacity of $p_{i}$ is created. And an edge is made from a vertex node $n$ to a skill node if the skill attributes of $n$ representing vertex contain the query skill represented by skill node. The capacity between $\mathrm{n}$ and skill node is set to the weight of that skill for that user.

Multiple algorithms exist in solving the maximum flow problem. Solving a min-cut problem for such successive flow networks can be done with time complexity equivalent to solving one of the min-cut problems. This technique for solving a maximum flow network problem is known as parametric flow network.[18].

\section{Experimental Study and results}

We conduct the experiments with real-world dataset to evaluate Geo-team formation model. We utilize edge density (ED) [8] and maximum communication cost [7] (MC) for Social cohesiveness evaluation. Furthermore we calculate keyword vertex percentage (KVP)[8] 
to evaluate skills relevance. To evaluate the Spatial closeness the spatial distance (Dist)[8] of result team to the team location $t$ is reported.

We use a real-world dataset in our experiments: Gowalla was a location-based social networking service [19]. Table 1 includes the statistical information of this dataset. In this dataset nodes without check-in information are deleted. And for nodes with multiple check-ins the first check-in is considered as the node location.

$$
\begin{array}{l|l|l|l|}
\text { Dataset } & \text { User } & \text { Edge } & \text { Avg.degree } \\
\text { Gowalla } & 107,092 & 456,830 & 27.81
\end{array}
$$

Table 1. The statistical information of utilized real-world dataset

To show the effectiveness of the Geo-team formation search, we consider two queries on the Gowalla dataset. The setting values of the queries are described as follows. On the first Query: team location $\mathrm{t}(-38.6,56.11)$, skills $\mathrm{N}=\mathrm{PHP}$, CSS, HTML, number of skills $\mathrm{O}=5,5,5$, contribution capacity $\mathrm{k}=3$, and the social constraints $\mathrm{p}=4$. In this query, we are looking for a web developer team to develop a website with PHP. For the second query, the value set is as follows: team location $\mathrm{t}(-38.6,56.11)$, skills $\mathrm{N}=$ piano, guitar, violin, number of skills $\mathrm{O}=1,1,2$, contribution capacity $\mathrm{k}=1$, and the social constraints $\mathrm{p}=4$. In the second query, the goal is to create a music band.

except for geo-team formation model, the geo-social group model MKASG in [20] is considered. The model's result is compared based on some used social cohesiveness and Spatial closeness metrics. In social cohesiveness, two metrics are calculated, the maximum communication cost [7] (MC) which is calculated based on the length of the maximum of the shortest path between all nodes of the resulting subgraph S. And the Edge Density [8] (ED), is evaluated based on $|\mathrm{E}(\mathrm{S})| /|\mathrm{V}(\mathrm{S})|$. Furthermore, for spatial closeness, the Dist $[8]$ is calculated based on the maximum Euclidean distance of team location $t$ and the subgraph $\mathrm{S}$ nodes. The comparisons of models on various metrics are depicted in Table 2.

\begin{tabular}{|l|l|l|l|l|} 
Model & Query & MC & ED & Dist \\
MKASG & Query 1 & 0.67 & 0.25 & 0.11 \\
Geo-team formation & Query 1 & 0.4 & 0.72 & 0.23 \\
MKASG & Query 2 & 0.54 & 0.28 & 0.13 \\
Geo-team formation & Query 2 & 0.25 & 0.76 & 0.5
\end{tabular}

Table 2. Effectiveness Evaluation

\section{Conclusion}

The Team Formation in Geo-Social Networks for impromptu activities tackles the problem of finding a team of experts from a Geo-social network who are socially and spatially close and cover the required team required skills. To solve this problem, we propose a novel model considering multiple constraints. Our preliminary results show considerable improvements to State-of-Art methods. We are planning to bring the concept of dynamic skill sets with the skill similarity score function in the future.

Acknowledgement. I want to express my great appreciation to Dr. Ziad Kobti for his valuable and constructive suggestions during this research work's planning and development. I would also like to thank Dr. Kalyani Selvarajah for her advice and assistance in keeping my schedule progress. 


\section{References}

[1] T. Lappas, K. Liu, and E. Terzi. "Finding a team of experts in social networks". In: Proceedings of the 15th ACM SIGKDD international conference on Knowledge discovery and data mining. 2009, pp. 467-476.

[2] C.-Y. Shen, D.-N. Yang, W.-C. Lee, and M.-S. Chen. "Spatial-proximity optimization for rapid task group deployment". In: ACM Transactions on Knowledge Discovery from Data (TKDD) 10.4 (2016), pp. 1-36.

[3] D. Papadias, Q. Shen, Y. Tao, and K. Mouratidis. "Group nearest neighbor queries". In: Proceedings. 20th International Conference on Data Engineering. IEEE. 2004, pp. 301-312.

[4] R Tao, T Habu, A Namba, H Yamane, F Fuyuhiro, K Iwamoto, and A Sugiura. "Inheritance of S f-RNase in Japanese apricot (Prunus mume) and its relation to self-compatibility". In: Theoretical and applied Genetics 105.2-3 (2002), pp. 222-228.

[5] N. Armenatzoglou, S. Papadopoulos, and D. Papadias. "A general framework for geo-social query processing". In: Proceedings of the VLDB Endowment 6.10 (2013), pp. 913-924.

[6] T. Lappas, K. Liu, and E. Terzi. "Finding a team of experts in social networks". In: Proceedings of the 15th ACM SIGKDD international conference on Knowledge discovery and data mining. 2009, pp. 467-476.

[7] C.-Y. Shen, D.-N. Yang, L.-H. Huang, W.-C. Lee, and M.-S. Chen. "Socio-spatial group queries for impromptu activity planning". In: IEEE Transactions on Knowledge and Data Engineering 28.1 (2015), pp. 196-210.

[8] L. Chen, C. Liu, R. Zhou, J. Xu, J. X. Yu, and J. Li. "Finding Effective Geo-social Group for Impromptu Activities with Diverse Demands". In: Proceedings of the 26th ACM SIGKDD International Conference on Knowledge Discovery \& Data Mining. 2020, pp. 698-708.

[9] Z. Li, Y. Lu, W.-P. Zhang, R.-H. Li, J. Guo, X. Huang, and R. Mao. "Discovering hierarchical subgraphs of k-core-truss". In: Data Science and Engineering 3.2 (2018), pp. 136-149.

[10] W. A. Gamson. "A theory of coalition formation". In: American sociological review (1961), pp. 373-382.

[11] B. Horling and V. Lesser. "A survey of multi-agent organizational paradigms". In: Knowledge Engineering Review 19.4 (2004), pp. 281-316.

[12] T. Rahwan, T. P. Michalak, M. Wooldridge, and N. R. Jennings. "Coalition structure generation: A survey". In: Artificial Intelligence 229 (2015), pp. 139-174.

[13] S. Liemhetcharat and M. Veloso. "Weighted synergy graphs for effective team formation with heterogeneous ad hoc agents". In: Artificial Intelligence 208 (2014), pp. 41-65.

[14] M. Husáková. "Immunity-Based Multi-Agent Coalition Formation for Elimination of Oil Spills". In: Soft Computing Models in Industrial and Environmental Applications. Springer, 2013, pp. 505-514.

[15] K. Taywade. "Multi-agent Reinforcement Learning for Decentralized Coalition Formation Games". In: (2021).

[16] Y. Fang, R. Cheng, X. Li, S. Luo, and J. Hu. "Effective community search over large spatial graphs". In: Proceedings of the VLDB Endowment 10.6 (2017), pp. 709-720.

[17] R. E. Tarjan. "Efficiency of a good but not linear set union algorithm". In: Journal of the ACM (JACM) 22.2 (1975), pp. 215-225.

[18] G. Gallo, M. D. Grigoriadis, and R. E. Tarjan. "A fast parametric maximum flow algorithm and applications". In: SIAM Journal on Computing 18.1 (1989), pp. 30-55.

[19] E. Cho, S. A. Myers, and J. Leskovec. "Friendship and mobility: user movement in locationbased social networks". In: Proceedings of the 17th ACM SIGKDD international conference on Knowledge discovery and data mining. 2011, pp. 1082-1090.

[20] Q. Zhu, H. Hu, C. Xu, J. Xu, and W.-C. Lee. "Geo-social group queries with minimum acquaintance constraints". In: The VLDB Journal 26.5 (2017), pp. 709-727. 